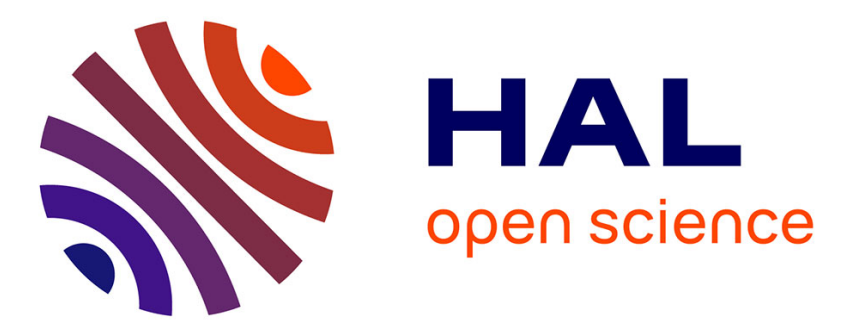

\title{
Pore network model of electrokinetic transport through charged porous media.
}

\author{
Amaël Obliger, Marie Jardat, Daniel Coelho, Samir Bekri, Benjamin \\ Rotenberg
}

\section{- To cite this version:}

Amaël Obliger, Marie Jardat, Daniel Coelho, Samir Bekri, Benjamin Rotenberg. Pore network model of electrokinetic transport through charged porous media.. Physical Review E: Statistical, Nonlinear, and Soft Matter Physics, 2014, 89 (4), pp.article No 043013. 10.1103/PhysRevE.89.043013 . hal01025807

\section{HAL Id: hal-01025807 \\ https://hal-ifp.archives-ouvertes.fr/hal-01025807}

Submitted on 22 Jul 2014

HAL is a multi-disciplinary open access archive for the deposit and dissemination of scientific research documents, whether they are published or not. The documents may come from teaching and research institutions in France or abroad, or from public or private research centers.
L'archive ouverte pluridisciplinaire HAL, est destinée au dépôt et à la diffusion de documents scientifiques de niveau recherche, publiés ou non, émanant des établissements d'enseignement et de recherche français ou étrangers, des laboratoires publics ou privés. 


\title{
Pore network model of electrokinetic transport through charged porous media
}

\author{
Amaël Obliger, ${ }^{1,2,3}$ Marie Jardat, ${ }^{1,2}$ Daniel Coelho, ${ }^{3}$ Samir Bekri, ${ }^{4}$ and Benjamin Rotenberg ${ }^{1,2}$ \\ ${ }^{1}$ Sorbonne Universités, UPMC Univ. Paris 06, UMR 8234 PHENIX, 75005 Paris, France \\ ${ }^{2}$ CNRS, UMR 8234 PHENIX, 75005 Paris, France \\ ${ }^{3}$ Andra, Parc de la Croix-Blanche, 1-7, rue Jean-Monnet, 92298 Châtenay-Malabry cedex, Franc $\AA^{*}$ \\ ${ }^{4}$ IFP Energies nouvelles, 1-4 Avenue de Bois-Préau, Rueil-Malmaison 92852, France
}

\begin{abstract}
We introduce a method for the numerical determination of the steady-state response of complex charged porous media to pressure, salt concentration and electric potential gradients. The macroscopic fluxes of solvent, salt and charge are computed within the framework of a Pore Network Model (PNM), which describes the pore structure of the samples as networks of pores connected to each other by channels. The PNM approach is used to capture the couplings between solvent and ionic flows which arise from the charge of the solid surfaces. The microscopic transport coefficients on the channel scale, taken here of a simple analytical form obtained previously by solving the Poisson-Nernst-Planck and Stokes equations in a cylindrical channel, are upscaled for a given network by imposing conservation laws for each pores, when macroscopic gradients are applied to the sample. The complex pore structure of the material is captured by the distribution of channel diameters. We investigate the combined effects of this complex geometry, the surface charge and the salt concentration on the macroscopic transport coefficients. The upscaled numerical model preserves the Onsager relations between the latter, as expected. The calculated macroscopic coefficients behave qualitatively like their microscopic counterparts, except for the permeability and the electro-osmotic coupling coefficient when the electrokinetic effects are strong. Quantitatively, the electrokinetic couplings increase the difference between the macroscopic coefficients and the microscopic ones for a single channel of average diameter.
\end{abstract}

\section{INTRODUCTION}

In charged porous media, the coupling between different transport phenomena arises from the excess electric charge of the fluid which compensates that of the solid walls. This charge may result both in the acceleration of the whole fluid under an applied electric field (electro-osmosis) and in the transport of charge if the fluid flows under an applied pressure gradient (streaming potential). In geophysics, the electroseismic effect, by which an electro-magnetic wave is generated from the motion of underground fluids under an applied acoustic wave, is exploited to determine the properties of geological formations [1]. Streaming potentials and electroosmotic flows can be measured in the laboratory to characterize the properties of porous media 4]. Electrokinetic phenomena also play an important role in biology [5] 7], membrane technology [8, 9], microfluidics [1012 with electrokinetic pumps [13] and more recently nanofluidics [14].

From the modelling perspective, upscaling the usual electrokinetic transport equations such as PoissonNernst-Planck (PNP) and Navier-Stokes (NS), from the microscopic scale where the couplings occur, to that of a macroscopic sample on which most experiments are performed, is a challenging task. Recently, significant progress has been made in this direction [15 17]. From the mathematical point of view, this upscaling can be performed rigorously using the homogenization approach [18 20]. The resulting set of coupled partial differential equations must then be solved numerically for a given cell representing the small scale of the porous medium. For practical applications, most studies of electrokinetic couplings rely on an oversimplified idealization of the geometry, with single slit pores or cylinders with dimensions or surface charge densities estimated from the macroscopic properties of the real system [21, 22]. However, the heterogeneity of the material, combined with the electrokinetic couplings, may influence the overall behaviour on the sample sacle, so that such idealizations may not reflect the actual response of the medium. Direct numerical resolution of the coupled PNP and NS equations in various complex systems (random packings, reconstructed and fractured porous media) has also been proposed by Adler and co-workers [23 25]. Such an approach is usually difficult to implement for macroscopic samples, due to the lack of experimental data on the fine structure of the material over large distances. The computational cost of direct numerical simulation also prevents the systematic study of a representative number of samples.

In the present paper, we introduce an alternative numerical scheme allowing for the description of transport through macroscopic charged porous materials at low computational cost, thereby enabling the systematic study of the combined effects of electrokinetic couplings and sample heterogeneity. Of course, such a goal can be achieved only at the price of simplifying the description. The proposed algorithm to upscale the electrokinetic couplings is based on the Pore Network Model (PNM). Such a model, originally developed by Fatt [26] to predict multiphase flow properties in porous media, describes the porosity as a network of pores connected by channels. During the last decades, it has been extensively used and 
extended by petrophysicists in various situations: capillarity and multiphase flow through porous media [27-31], pore evolution and changes in petrophysical properties due to particle capture 32, filtration combustion 33, mineral dissolution and precipitation caused by $\mathrm{CO}_{2}$ sequestration [34] and adsorption/reaction processes [35]. Recently, Varloteaux et al. 36] studied the reactive transport in porous media using a PNM. Bernabé et al. [37] proposed a PNM approach for the streaming potential in geophysics based on the description of the electrokinetic effects in porous media of 38 .

In a nutshell, the PNM approach amounts to solving a set of conservation equations on the nodes of the network (in analogy with Kirchhoff's law for a network of resistors), on the basis of local fluxes through the channels connecting the nodes, under the effect of an external, macroscopic gradient. In most of the cases treated to date (see [36] for an exception), the solution to this problem is a linear one. On the channel scale, the description of the electrokinetic transport used here follows the approach of Rice and Whitehead [39] and allowed us to derive the longitudinal ionic and solvent flows through an infinite charged cylinder driven by a gradient of pressure, salt concentration or electric potential [40. We take advantage here, for the determination of the fluxes through each channel, from the observation of Gupta et al. 41] that the electrokinetic effects in non-trivial geometries can be captured by a simpler one, provided that the problem on the pore scale is renormalized by an appropriate length scale, initially introduced by Johnson et al. [42. The transport coefficients through each channel depend here on the properties of the pores at both ends, leading to a non-linear structure of the macroscopic problem. The objective is then two-fold: On the one hand, to introduce the numerical scheme for the upscaling of the electrokinetic transport equations with the PNM approach; on the other hand, to investigate how the upscaled properties depend on the distribution of channel diameters inside the material, in addition to the surface charge density and the salt concentration.

The paper is organized as follows. In Sec. II , we first recall the description of coupled transport phenomena in porous media on the macroscopic (sample) and microscopic (pore) scales. The homogenization procedure used to upscale these quantities is presented in Sec. III, as is the construction of the network realizations from channel diameter distributions. Finally, all macroscopic transport coefficients, determined numerically as a function of the salt concentration and the surface charge density, are presented in Sec. IV for heterogeneous samples. We discuss the influence of the channel diameter distribution on the macroscopic properties.

\section{ELECTROKINETIC TRANSPORT THROUGH CHARGED POROUS MEDIA}

\section{A. Pore Network Model of charged porous media}

We consider here the linear response of a macroscopic sample, initially in equilibrium with two solution reservoirs, to macroscopic pressure, salt concentration and electric potential gradients, which result in solvent and monovalent cations and anions (or equivalently, salt concentration and electric charge) flows. On the macroscopic scale, the steady state total flows of solvent, $Q_{0}$, salt, $Q_{1}$, and charge, $Q_{2}$, through the network are proportional to the macroscopic pressure, $P$, salt chemical potential, $C$ (see below), and electric potential, $V$ gradients. The aim of the present work is thus to determine the macroscopic transfer matrix $K$, defined by:

$$
\left(\begin{array}{l}
Q_{0} \\
Q_{1} \\
Q_{2}
\end{array}\right)=-\frac{L_{n}^{2}}{\eta}\left(\begin{array}{ccc}
K_{0}^{P} & K_{0}^{C} & K_{0}^{V} \\
K_{1}^{P} & K_{1}^{C} & K_{1}^{V} \\
K_{2}^{P} & K_{2}^{C} & K_{2}^{V}
\end{array}\right)\left(\begin{array}{c}
\nabla P \\
\nabla C \\
\nabla V
\end{array}\right)
$$

with $L_{n}$ the total width of the sample (hence the distance between the two solution reservoirs) and $\eta$ the fluid viscosity. This matrix should be symmetric, as a result of Onsager's reciprocity principle [20, 43].

As previously mentioned, the PNM consists of a lattice of pores connected to each other by channels. The pores are characterized only by their fluid pressure, salt concentration and electric potential, while the channels are characterized by their diameter and their surface charge density. The transfer properties of each channel depend on the amount of salt inside it, which in turn is controlled by the Donnan equilibrium existing between the channel and the pores (or, equivalently at equilibrium, between the channel and external reservoirs). In the linear response regime, the solvent and ionic flows are proportional to the local gradients and thus determined by the transfer (or conductance) matrix of each channel, which may be determined by solving the underlying transport equations on the channel scale. Figure 1 illustrates the main features of this pore network model (PNM).

The central ingredient of a PNM is the conductance (here conductance matrix) of the channels, not the actual geometry of the medium. Note that no geometrical features of the pores enters in the present model: their effect on the flow is entirely encompassed in their indirect effect on the channels (Donnan equlibrium which influences the conductance matrix of the channel and gradient across the channel). In addition, the conductance matrix of each channel (see below) is computed for an infinite cylindrical channel. In particular, the pores do not have a definite size and the converging-diverging nature of real pores [44, 45] is not taken into account explicitly. This can be interpreted as assuming a separation of length scales between the pore and channel characteristic 


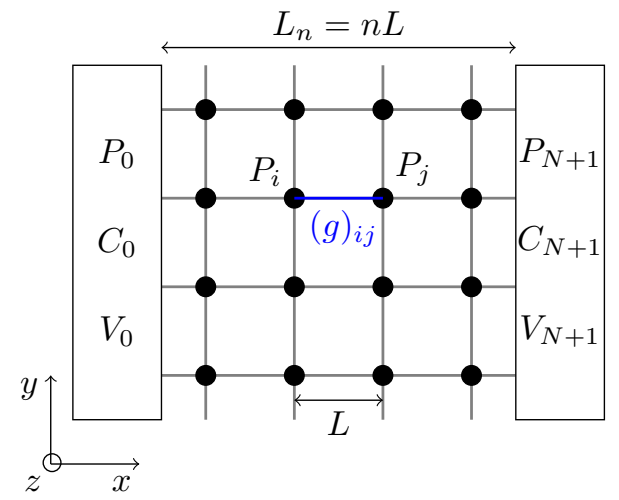

FIG. 1: Two-dimensional representation of a cubic network consisting of $N=n \times n \times n$ pores, between two reservoirs, labelled 0 and $N+1$. Each pore is characterized by a pressure $P_{i}$, a salt concentration $c_{i}$ leading to an ideal part of the chemical potential $C_{i}=k_{B} T \ln \left(c_{i} / c_{N+1}\right)$, and an electric potential $V_{i}$. Two connected pores $i$ and $j$ (denoted $i \backsim j$ ) are separated by a length $L$ (hence a total width of $L_{n}=n L$ ). The flows of solvent, salt and charge between these pores under the effect of pressure, salt concentration and potential gradients is, in the linear response regime, determined by the transfer (or conductance) matrix $(g)_{i j}$, which depends on the surface charge density and on the salt concentration inside the corresponding channel. The latter is governed by the Donnan equilibrium between the channel and the pores.

lengths. Note that it is possible to introduce pore sizes in a PNM, at the price of introducing short-range correlations between the pore and channel diameters 46, 47.

In the following, we address the combined role of the channel size distribution inside the sample, which may be very heterogeneous, as well as that of the surface charge density of the solid and of the salt concentration in the reservoirs, on the transfer matrix. Before turning to the upscaling from the channel to the sample scale, we first recall the influence of the channel diameter, the surface charge density and the salt concentration, on the transfer properties of a single channel.

\section{B. Transfer matrix on the channel scale}

The linear response of a channel $i j$ between two pores $i$ and $j$ follows the same structure as the macroscopic reponse (1). The local flows of solvent, $q_{0}$, salt, $q_{1}$, and charge (electric current), $q_{2}$ are written as:

$$
\left(\begin{array}{l}
q_{0} \\
q_{1} \\
q_{2}
\end{array}\right)_{i j}=-\frac{\pi d^{2}}{4 \eta}\left(\begin{array}{lll}
g_{0}^{P} & g_{0}^{C} & g_{0}^{V} \\
g_{1}^{P} & g_{1}^{C} & g_{1}^{V} \\
g_{2}^{P} & g_{2}^{C} & g_{2}^{V}
\end{array}\right)_{i j}\left(\begin{array}{c}
\nabla_{i j} P \\
\nabla_{i j} C \\
\nabla_{i j} V
\end{array}\right),
$$

where $d$ is the pore diameter and where the gradient of pore variables are defined as

$$
\nabla_{i j} X=\frac{X_{j}-X_{i}}{L}
$$

with $L$ the distance between pore centers. $L$ is also the length of the channels. $P_{i}$ is the pressure, $C_{i}=$ $k_{B} T \ln \left(c_{i} / c_{N+1}\right)$ is the salt chemical potential and $V_{i}=$ $\psi_{i}-\psi_{N+1}$ the electric potential. The salt concentration $c_{i}$ in pore $i$ enters in the ideal chemical potential together with a reference concentration, taken as the one in the reservoir in the absence of perturbing gradient. In the following, we will assume without loss of generality that gradients are imposed by changing the properties of the left reservoir (labelled 0, see Figure 1). The right reservoir (labelled $N+1$ ) is then used as the reference for the salt concentration and electric potential. Hence $C_{N+1}=V_{N+1}=0$. The absolute value of the pressure does not play a role and we also set by convenience $P_{N+1}=0$.

The coefficients of the channel transfer matrix defined by (2) can be determined by examining the mechanisms underlying the coupling between the solvent and ionic flows. In principle the coefficients could be determined with any method, including numerically from molecular simulations, as a function of the relevant parameters. The standard description of these coupled solvent and ionic flows rely on the solution of a set of coupled differential equations, namely: (a) Navier-Stokes, which accounts for momentum conservation inside the fluid and captures the effect of all local forces, including the electric force arising from the local electric charge and electric field; (b) Nernst-Planck, which accounts for the conservation of ions and includes the effect of advection by the fluid flow; (c) Poisson, which determines the electric potential distribution arising from the charge distribution. The fluxes of solvent and ions under pressure, salt concentration and electric potential gradients can then be solved for a given channel geometry and boundary conditions at its surface.

Here we consider for simplicity the case of infinite cylindrical channels of diameter $d$ with a fixed, homogeneous surface charge density $\sigma$ (in $e . n m^{-2}$ ), with a solvent of viscosity $\eta$ and permittivity $\epsilon$, and monovalent cations and anions with diffusion coefficients $D_{ \pm}$, at a temperature $T$. While the cylindrical geometry seems far from a realistic representation of a complex porous medium, one should keep in mind that within a PNM approach it is the channel conductance (matrix) which is relevant, not its geometry. It has been shown previously by Gupta et al. that the transfer properties through more complex channels can be captured by a simple expression for the cylindrical geometry, provided that a correct renormalizing length scale $(\Lambda)$ is used 41 . Under the assumption of small potential differences between the walls and the solution, the Poisson equation can be linearized and the set of equations solved analytically for this geometry. We have previously validated these approximate results against numerical simulation in the range of parameters used here [40]. Outside the range of validity of this assumption, e.g. for large surface charge densi- 
ties, the coefficients can be computed numerically and introduced in the present PNM.

More precisely, the PNM requires the knowledge of the flows of solvent, $q_{0}$, salt, $q_{1}$, and charge (electric current), $q_{2}$, which in the case of cylindrical channel read:

$$
q_{\alpha}^{\gamma}=\int_{0}^{d / 2} j_{k}^{\gamma} 2 \pi r \mathrm{~d} r
$$

where $j_{0}=u$ is the local solvent velocity, $j_{1}=j_{+}+j_{-}$ and $j_{2}=e\left(j_{+}-j_{-}\right)$, with $j_{+}$(resp. $\left.j_{-}\right)$the local cation (resp. anion) flux. The superscript $\gamma=(P, C, V)$ refers to the applied forcing, namely a pressure, concentration (in fact, salt chemical potential) and potential gradient, respectively.

Under the above assumptions, the coefficients of the transfer matrix defined by (2) are as follows (the channel label $i j$ is omitted for clarity). For the solvent under a pressure gradient one finds the usual permeability (note the $\pi d^{2} / 4 \eta$ prefactor in Eq. (2)):

$$
g_{0}^{P}=\frac{d^{2}}{32} .
$$

Under a concentration gradient, the osmotic term which by symmetry corresponds to the salt flux under a pressure gradient is

$$
g_{0}^{C}=g_{1}^{P}=\frac{\kappa^{2} d^{2}}{128 \pi l_{B}},
$$

where $\kappa^{2}=4 \pi l_{B}\left(c^{+}+c^{-}\right)$is the square of the inverse Debye screening length, with $l_{B}=\beta e^{2} / 4 \pi \epsilon$ the Bjerrum length $\left(\beta=1 / k_{B} T\right)$ and $c^{+}$(resp. $\left.c^{-}\right)$the average cation (resp. anion) concentrations in the channel, to be discussed below. Under an applied electric field the electro-osmosic solvent flux (by symmetry, it also governs the streaming current) is given by:

$$
g_{0}^{V}=g_{2}^{P}=-\frac{4 \sigma}{\kappa} \frac{\mathrm{I}_{2}}{\mathrm{I}_{1}}
$$

where $\mathrm{I}_{m}=\mathrm{I}_{m}(\kappa d / 2)$ are modified Bessel functions of the first kind. The salt flux under a concentration gradient follows from

$$
g_{1}^{C}=\beta \eta\left(D_{+} c^{+}+D_{-} c^{-}\right)+\frac{\kappa^{4} d^{2}}{512 \pi^{2} l_{B}^{2}},
$$

with a diffusive part (Fick) and an advective part due to osmosis. Under an applied electric field, the motion of ions contains a direct (Nernst-Einstein) contribution and an advective part due to electro-osmosis, resulting in

$$
g_{1}^{V}=g_{2}^{C}=e \beta \eta\left(D_{+} c^{+}-D_{-} c^{-}\right)-\frac{\sigma \kappa}{4 l_{B}} \frac{\mathrm{I}_{2}}{\mathrm{I}_{1}}
$$

for the flux of salt and in

$$
g_{2}^{V}=e^{2} \beta \eta\left(D_{+} c^{+}+D_{-} c^{-}\right)-\sigma^{2}\left[1-\frac{\mathrm{I}_{0} \mathrm{I}_{2}}{\mathrm{I}_{1}^{2}}\right],
$$

for the electric current. The symmetry of the transfer matrix is explicit in Eqs. (6) and (7) for the solute and electric current arising from a pressure gradient, and in Eq. (9) for the electric current under a concentration gradient.

\section{Donnan equilibrium}

It appears from the previous section that the coefficients of the channel $i j$ transfer matrix $(g)_{i j}$ depend on the average ionic concentrations $c_{i j}^{+}$and $c_{i j}^{-}$inside the channel. As we consider the linear response around an equilibrium situation, these concentrations can be determined by considering the (Donnan) equilibrium of the ions between the charged channel and the adjacent pores seen as an equivalent reservoir. Writing the equality of ionic chemical potentials in the channel and in the reservoir, one obtains:

$$
\beta\left(\mu_{i j}^{ \pm}-\mu_{\text {res }}^{ \pm}\right)=\ln \frac{c_{i j}^{ \pm}}{c_{\text {res }}} \pm \beta e\left(V_{i j}-V_{\text {res }}\right)=0,
$$

with $V_{i j}$ the so-called Donnan potential, and $c_{\text {res }}=\sqrt{c_{i} c_{j}}$ (resp. $V_{\text {res }}=\left(V_{i}+V_{j}\right) / 2$ ) the salt concentration (resp. potential) in the electrically neutral reservoir. Noting that the excess of counterions with respect to co-ions balances that of the surface, i.e. $c_{i j}^{+}-c_{i j}^{-}=c_{i j}^{\sigma}=-4 \sigma / e d_{i j}$ in a cylindrical channel with diameter $d_{i j}$, the two equalities (11) straitforwardly lead to:

$$
c_{i j}^{ \pm}=\sqrt{\left(c_{\mathrm{res}}\right)^{2}+\left(\frac{c_{i j}^{\sigma}}{2}\right)^{2}} \pm \frac{c_{i j}^{\sigma}}{2} .
$$

The ionic concentrations in the channel can thus be computed from the channel diameter $d_{i j}$, the surface charge density $\sigma$ and the salt concentration in the reservoir with which the channel is in equilibrium. Once the salt concentration in the channel is known, all the transport coefficients can be computed as described in the previous section.

\section{TRANSPORT ON THE MACROSCOPIC SCALE}

\section{A. Determination of the transport coefficients}

The macroscopic transfer matrix $(K)$ defined by Eq. (1) can now be determined for a given network, characterized by the diameter $d_{i j}$ of each channel and the corresponding transfer matrix $(g)_{i j}$ - keeping in mind that the latter also depends on the surface charge density and on the reservoir salt concentration. To that end, macroscopic gradients are imposed through the network by changing the pressure, salt concentration or electric 
potential of the left reservoir (the right one being maintained at the initial values). The steady state local fluxes are then computed by ensuring the conservation of solvent, salt and charge in each pore $i$, i.e.:

$$
\sum_{i \backsim j}\left(\begin{array}{l}
q_{0} \\
q_{1} \\
q_{2}
\end{array}\right)_{i j}=\mathbf{0}
$$

This provide a set of non-linear equations for the pore variables $\left\{P_{i}\right\},\left\{C_{i}\right\}$ and $\left\{V_{i}\right\}$ at steady state. The nonlinearity arises from the fact that the coefficients of the matrix in (2) depend on the pore variables via the local Donnan equilibrium. In practice, we solve this system of equations numerically using a non-linear Newton solver described in [48. Periodic boundary conditions are used in the directions perpendicular to the macroscopic gradient. The macroscopic fluxes through the sample are finally computed at the interface with either reservoir:

$$
\left(\begin{array}{l}
Q_{0} \\
Q_{1} \\
Q_{2}
\end{array}\right)=\sum_{j_{0}}\left(\begin{array}{l}
q_{0} \\
q_{1} \\
q_{2}
\end{array}\right)_{0, j_{0}}=\sum_{j_{N+1}}\left(\begin{array}{l}
q_{0} \\
q_{1} \\
q_{2}
\end{array}\right)_{i_{N+1}, N+1}
$$

where $j_{0}$ (resp. $\left.i_{N+1}\right)$ labels all the pores connected to the left (resp. right) reservoir. Evaluating the coefficients of the matrix $K$ requires solving 3 times the system of non-linear equations (13) for the pore variables, corresponding to the three macroscopic gradients (pressure, concentration and potential). Each macroscopic gradient induces 3 macroscopic flows (solvent, salt and charge), thereby providing the 9 coefficients. As an example, the first column of the matrix $K$ is computed by imposing only a macroscopic pressure gradient (i.e. $\nabla C=0$ and $\nabla V=0)$ as:

$$
K_{\alpha}^{P}=-\frac{\eta Q_{\alpha}}{L_{n}^{2} \nabla P}, \quad \alpha=0,1,2
$$

For $\alpha=0$ this is simply Darcy's law. The macroscopic matrix $K$ is expected to be symmetric [20, 43, regardless of the diameter distribution inside the network, a property which is verified in our numerical simulations. Finally, it is worth noting that the overall balance of local fluxes may be achieved by the compensation of fluxes induced by local gradients of different nature than the macroscopic one (e.g. a local electric field in the absence of macroscopic potential difference).

\section{B. Statistical properties of pore networks}

The previous sections allow us to determine the transfer matrix $K$ for a given network. When studying porous materials, an important question is that of the representativity of a given model. If detailed geometric information is available from appropriate imaging techniques, an equivalent network can be built for a given experimental sample. Most often, however, only indirect information such as a pore size distribution is available experimentally. In that case, the transfer matrix of a single network is not sufficient to describe the macroscopic properties. Rather, one should consider the statistical properties of the transfer matrix over networks drawn from the available (or model) size distribution. In particular, we investigate here how the macroscopic coefficients depend on the mean and standard deviation of the channel diameter distribution.

We thus consider here the properties of ensemble of networks characterized by a uniform temperature, fluid viscosity and permittivity, surface charge density, ion diffusion coefficients, and channel length but with varying channel diameters. More precisely, the channel diameters $d_{i j}$ are drawn randomly from a Weibull distribution function,

$$
f\left(d ; d_{m i n}, k, \lambda\right)=\frac{k}{\lambda} g\left(\frac{d-d_{\min }}{\lambda}\right),
$$

with $g(x)=x^{k-1} e^{-x^{k}}, d_{\text {min }}$ the minimum diameter, and $k$ and $\lambda$ two positive parameters defining the shape of the distribution. They control in particular the mean diameter $\bar{d}=\lambda \Gamma(1+1 / k)$ and the variance $\delta^{2}=\lambda^{2} \Gamma(1+2 / k)-$ $\bar{d}^{2}$, where $\Gamma$ is the gamma function. The distributions investigated numerically in the following are illustrated in Fig. 2. For a given distribution function, we generate $M$ networks and compute the macroscopic transfer matrix. We finally determine the average coefficients over the $M$ samples (i.e. realizations of the distribution function) and the corresponding variances.

\section{Validation of the method}

We now turn to the numerical validation of the proposed algorithm. To that end, we consider values of the various physical parameters having in mind the case of clay minerals, which are typical examples of natural charged porous media. More specifically, we consider a system at $T=298 \mathrm{~K}$ with water as a solvent $\left(\eta=10^{-3}\right.$ Pas and $\left.\epsilon=78.5\right)$ with $\mathrm{NaCl}$ as monovalent salt $\left(D_{+}=1.310^{-9} \mathrm{~m}^{2} \mathrm{~s}^{-1}\right.$ and $\left.D_{-}=2.010^{-9} \mathrm{~m}^{2} \mathrm{~s}^{-1}\right)$. The surface charge density is taken in the range $\sigma \in$ $[-0.1,0] e \mathrm{~nm}^{-2}$, with $\mathrm{Na}^{+}$as a counterion, and salt concentrations in the range $c \in\left[10^{-3}, 10^{-1}\right] \mathrm{mol} \mathrm{L}^{-1}$. The distance between pore centers is $L=160 \mathrm{~nm}$ and the minimum channel diameter is $d_{\min }=4 \mathrm{~nm}$. While smaller pores can be found in clay minerals, the PNP model used here to describe the electrolyte in the channels is not accurate below this limit. Since the purpose of the present work is not to accurately describe a specific system but rather to demonstrate the relevance of the PNM approach to model complex charge porous materials, we simply restrict ourselves to situations where the 


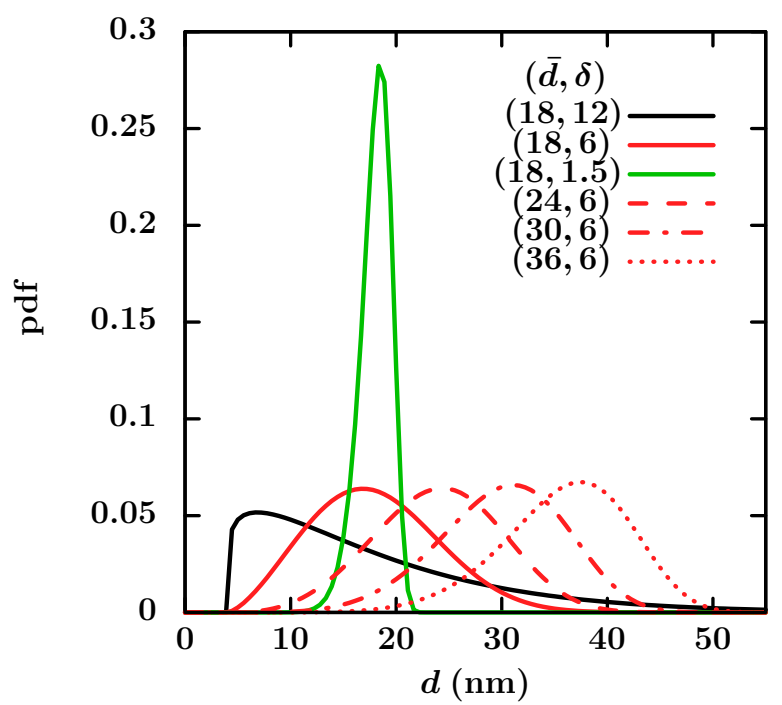

FIG. 2: (Color online) Weibull distribution functions used in this study to generate networks, labelled according to their mean diameter and standard deviation $(\bar{d}, \delta)$. Solid lines correspond to the same mean diameter $\bar{d}=18 \mathrm{~nm}$, while dashed and dotted lines correspond to the same standard deviation $\delta=6 \mathrm{~nm}$. The minimum diameter (see Eq. 16) is $d_{\text {min }}=4 \mathrm{~nm}$.

microscopic model on the channel scale applies. The extension of the PNM beyond its domain of validity is only limited by the availability of appropriate expressions for the conductance matrix of the channel.

We consider several values of the mean diameter $\bar{d}(18$, $24,30$ and $36 \mathrm{~nm})$ and standard deviation $\delta(1.5,6$ and $12 \mathrm{~nm})$. We found that for this range of parameters, a number $M=80$ samples to perform averages over networks for a given distribution functions provided a good compromise between accuracy and computational cost. This parameter will be kept constant throughout this paper. In order to remain in the linear response regime, which is the only one considered here, we apply small macroscopic gradients. More specifically, the results reported below were performed with $\nabla P=10^{-6}$ $\mathrm{Pa} \mathrm{nm}{ }^{-1}, \nabla C=10^{-9} \mathrm{~J} \mathrm{~nm}^{-1}$ and $\nabla V=10^{-14} \mathrm{~V} \mathrm{~nm}^{-1}$. Another consequence of the linear response is that the fluxes measured in the presence of several macroscopic gradients should be additive. We have checked that this is indeed verified numerically in all the considered cases (with relative errors smaller than $10^{-5 \%}$ ).

The last parameter which should be chosen carefully is the number $N=n^{3}$ of network nodes. This number should be sufficiently large to ensure a good sampling of the size distribution function. The measured average transfer matrix $K_{n}$ should converge for large network sizes to a limit $K_{\infty}$, which is the "true" average over the size distribution (note that one should also consider the limit of infinite number $M$ of samples). As an example of this convergence, results for the permeability $K_{0}^{P}$ as a func- tion of $n$ are reported in Figure 3, for a mean diameter $\bar{d}=18 \mathrm{~nm}$ and several values of the standard deviation $\delta$. For each network size $n$, the average and variance of the permeability over the $M$ samples are indicated. The average permeability converges toward a finite value $K_{\infty}$ for large $n$ and the variance of the distribution of $K$ around $K_{\infty}$ decreases as $n$ increases or as $\delta$ decreases, as expected. Note that in the simple case where all the channels are identical $(\delta=0 \mathrm{~nm})$, the macroscopic transfer matrix $K$ should be equal to that of each channel $g$. For the range of parameters given above, this is indeed the case with relative errors smaller than $10^{-5} \%$ for sizes $n<30$. In the following, we use $n=20$, which typically provides estimates of $K_{\infty}$ within $1 \%$. Such a network size is comparable to previsous PNM studies of other transport phenomena (see e.g. [46]).

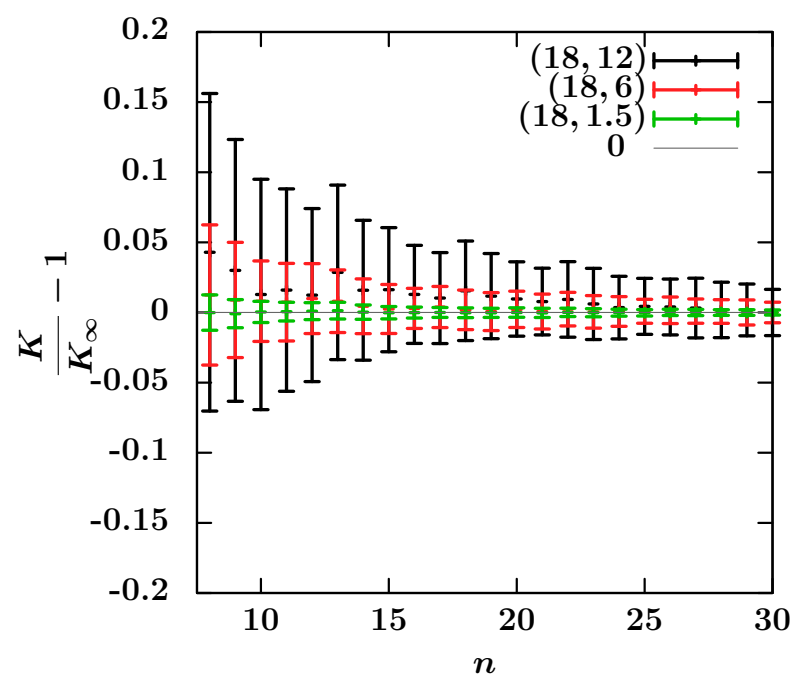

FIG. 3: (Color online) Permeability $K_{0}^{P}$ as a function of the network size $n$ for a surface charge density $\sigma=-0.1 \mathrm{enm}^{-2}$ and salt concentration in the reservoir $c=0.001 \mathrm{~mol} \mathrm{~L}^{-1}$. Results are reported compared to the limit $K_{\infty}$ (taken as the value for $n=30$ ), for diameter distributions of mean $\bar{d}=18 \mathrm{~nm}$ and standard deviation: $\delta=12 \mathrm{~nm}$ (black), $6 \mathrm{~nm}$ (red) and $1.5 \mathrm{~nm}$ (green). The average and variance are computed over $M=80$ realizations for each distribution. The results obtained with other values for $c$ and $\sigma$ are very similar.

\section{MACROSCOPIC TRANSFER MATRIX}

We now turn to the numerical study of the macroscopic transfer coefficients. We first investigate, for a given channel diameter distribution, the influence of the solid surface charge density and of the salt concentration in the reservoir. We then turn, for a fixed surface charge density, to the combined effect of diameter distribution and salt concentration. As explained above, for each distribution the reported results correspond to an average 
over several samples from this distribution.

\section{A. Influence of surface charge density and salt concentration}

We first study the influence of the salt concentration $c$ in the reservoirs and of the surface charge $\sigma$ for a Weibull distribution with a mean diameter $\bar{d}=18 \mathrm{~nm}$ and a standard deviation $\delta=12 \mathrm{~nm}$, with the other parameters taken as described in the previous section. The macroscopic transport matrix is symmetric, as expected, and we report its coefficients in the same way as the channel coefficients in Section IB.

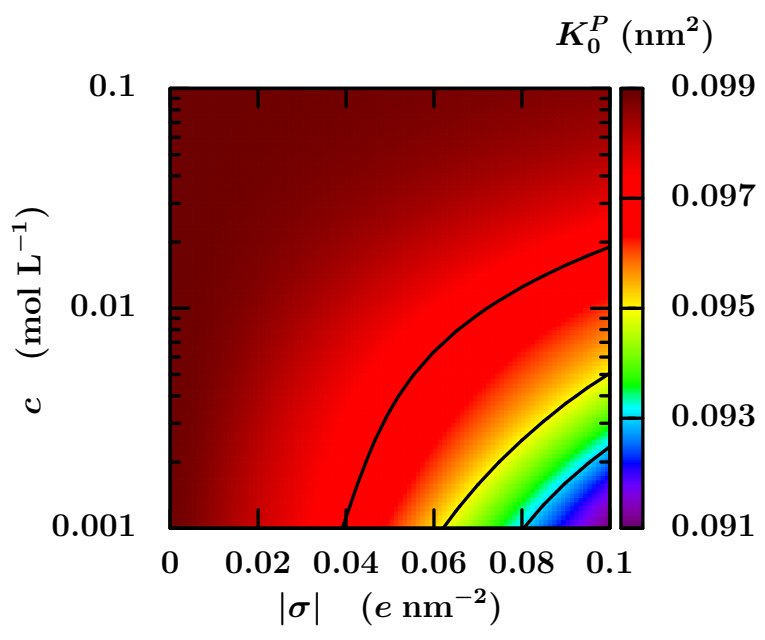

FIG. 4: (Color online) Macroscopic permeability $K_{0}^{P}\left(\right.$ in $\mathrm{nm}^{2}$ ) as a function of the salt concentration $c$ in the reservoirs in equilibrium with the charged porous material, and of the surface charge density $\sigma$ of the channels.

Fig. 4 reports the macroscopic permeability $K_{0}^{P}$. While for neutral walls $(\sigma=0)$ it is independent of the salt concentration $c$ in the reservoirs, $K_{0}^{P}$ decreases with increasing $|\sigma|$ or decreasing $c$. Such a result may seem surprising, because for a single channel the permeability (Eq. 5) does not depend on the presence of charges on the wall or in solution. This variation on the macroscopic scale is due to the fact that the macroscopic fluxes result from a balance between different local fluxes, including electrokinetic ones. As an example, a macroscopic pressure gradient induces local streaming currents which tend to separate charges locally. In turn, this results in local electric potential gradients across the channels which induce electro-osmotic flow, thereby mitigating the local pressure-driven solvent flow. Such electrokinetic couplings are more pronounced for large $|\sigma|$ and low $c$. For large salt concentrations (near $0.1 \mathrm{~mol} \mathrm{~L}^{-1}$ ), the screening of electrostatic interactions results in smaller elec- trokinetic effects, hence a smaller influence of the surface charge density on the macroscopic permeability. As will be discussed below, the coupling between the various types of local fluxes and the resulting decrease in the macroscopic permeability $K_{0}^{P}$ also depends on the heterogeneity of the material.

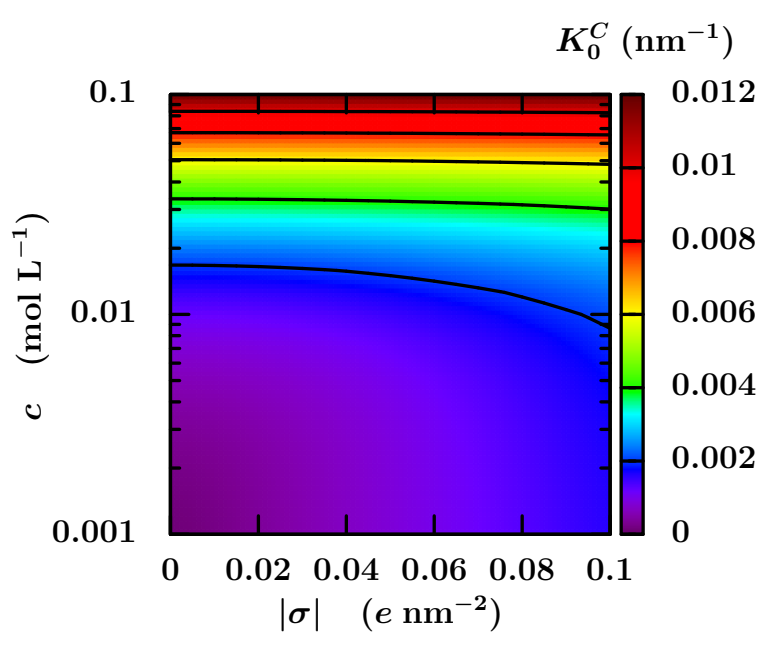

FIG. 5: (Color online) Macroscopic osmotic coefficient $K_{0}^{C}$ (in $\mathrm{nm}^{-1}$ ) as a function of the salt concentration $c$ in the reservoirs in equilibrium with the charged porous material, and of the surface charge density $\sigma$ of the channels.

The osmotic coefficient $K_{0}^{C}$, governing the solvent flow under a salt concentration gradient, is reported in Fig. 5 . As expected, the osmotic flux increases with the salt concentration. As observed for the permeability, the effect of the surface charge density is more pronounced for smaller salt concentrations. The evolution of $K_{0}^{C}$ with $c$ and $\sigma$ qualitatively follows that of the corresponding coefficient on the channel scale $g_{0}^{C}$ given by Eq. (6), discussed in [40]. From the symmetry of the macroscopic transfer matrix, these results also hold for the salt flux under a pressure gradient, quantified by the coefficient $K_{1}^{P}$.

Fig. 6 reports the electro-osmotic coefficient $K_{0}^{V}$, which quantifies the solvent flux under an electric potential gradient. When $\sigma=0$, this effect is absent; it increases with $|\sigma|$. As $g_{0}^{V}$ on the channel scale [40], the macroscopic coefficient $K_{0}^{V}$ decreases with increasing ionic strength as a result of the screening of the surface charge. At large surface charge densities, decreasing the salt concentration to very small values results in a slight decrease of $K_{0}^{V}$. This contrasts with the case of a single channel, given by Eq. (7), which plateaus in this limit (see also Fig. 11 below). Such a decrease is also a consequence of the balance between different types of local fluxes.

The coefficient $K_{1}^{C}$, reported on Fig. 7 quantifies the flow of solute under a macroscopic concentration gradient. This coefficient is mainly controlled by the salt con- 


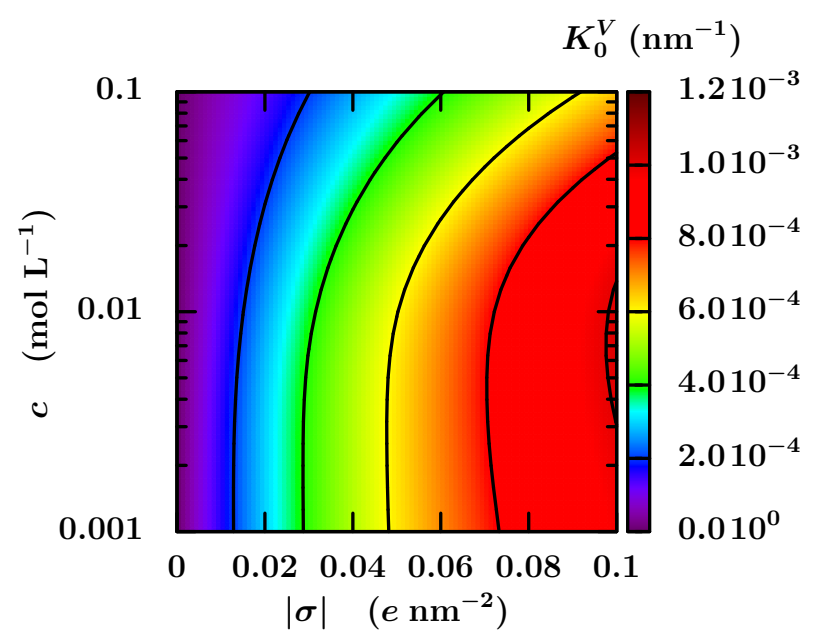

FIG. 6: (Color online) Macroscopic electro-osmotic coefficient $K_{0}^{V}$ (in $\mathrm{nm}^{-1}$ ) as a function of the salt concentration $c$ in the reservoirs in equilibrium with the charged porous material, and of the surface charge density $\sigma$ of the channels.

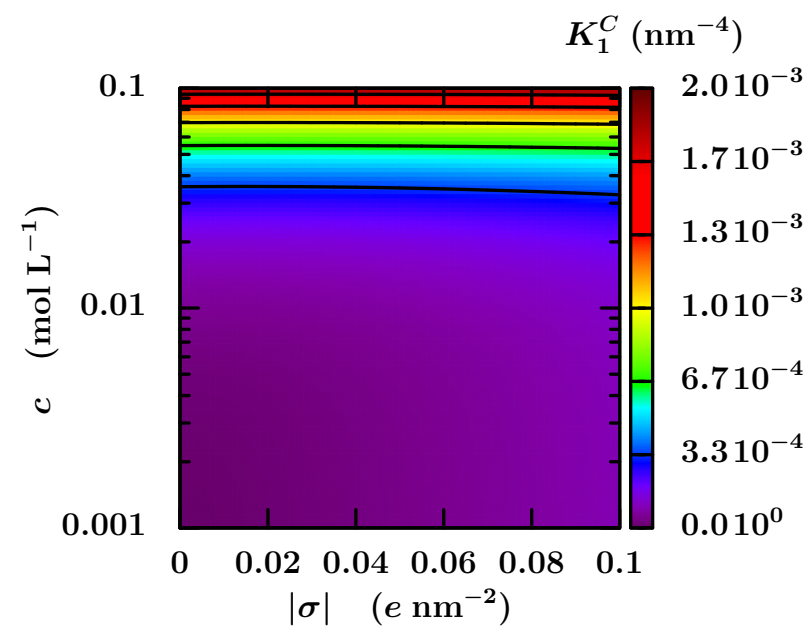

FIG. 7: (Color online) Macroscopic coefficient $K_{1}^{C}$ (in $\mathrm{nm}^{-4}$ ) as a function of the salt concentration $c$ in the reservoirs in equilibrium with the charged porous material, and of the surface charge density $\sigma$ of the channels.

centration $c$. It captures both the effect of diffusion and that of advection by the osmotic flow. For large surface charge densities, the latter slightly increases the overall solute flux, as does $g_{1}^{C}$ given by Eq. (8).

The effect of an electric field on the solute transport is quantified by $K_{1}^{V}$, reported in Fig. 8. A noticeable feature of this coefficient is the existence of two regions corresponding to negative and positive values. This is due to the different values of the diffusion coefficients for sodium cations and chloride anions considered here

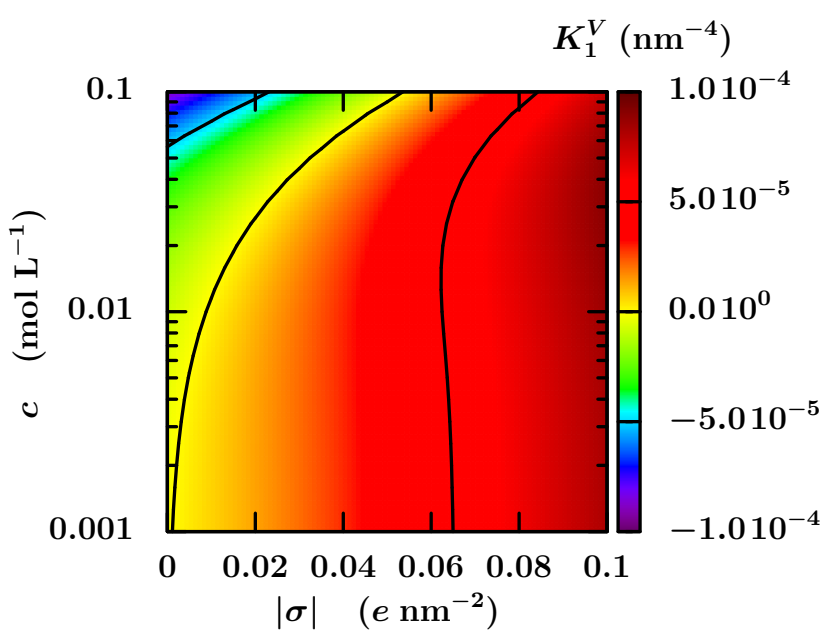

FIG. 8: (Color online) Macroscopic coefficient $K_{1}^{V}$ (in $\mathrm{nm}^{-4}$ ) as a function of the salt concentration $c$ in the reservoirs in equilibrium with the charged porous material, and of the surface charge density $\sigma$ of the channels.

$\left(D_{-}>D_{+}\right)$. Depending on their relative concentrations in the porous medium, which depend both on the surface charge density and on the salt concentration in the reservoirs, the overall salt flux can be either in the direction of the electric field or in the opposite one. Note that an improved description of the ionic transport inside the channels could capture the effect of internal fields stemming from the different ionic mobilities (Nernst-Hartley theory). At large surface charge densities, decreasing the salt concentration to very small values results in a slight decrease of $K_{1}^{V}$, as observed for $K_{0}^{V}$. In the present case, however, this decrease is already present at the channel level, i.e. $g_{1}^{V}$, given by Eq. (7).

Finally, Fig. 9 reports the macroscopic electrical conductance $K_{2}^{V}$. It increases with the salt concentration and with the surface charge density. The latter increase is more pronounced for lower salt concentrations. Here again, the variations of the macroscopic coefficient follow that of the microscopic one, $g_{2}^{V}$ given by Eq. 10 .

\section{B. Influence of the diameter distribution}

Overall, it appears that except for the permeability, the macroscopic coefficients behave qualitatively as the microscopic ones for a single channel of diameter $d=\bar{d}$, the average diameter. The deviations, including the qualitative difference for the permeability, arise from combined effect of the couplings between the various types of fluxes which must balance locally and of the heterogeneity of the sample (we recall here that for a network with identical channels the macroscopic coefficients coincide with 


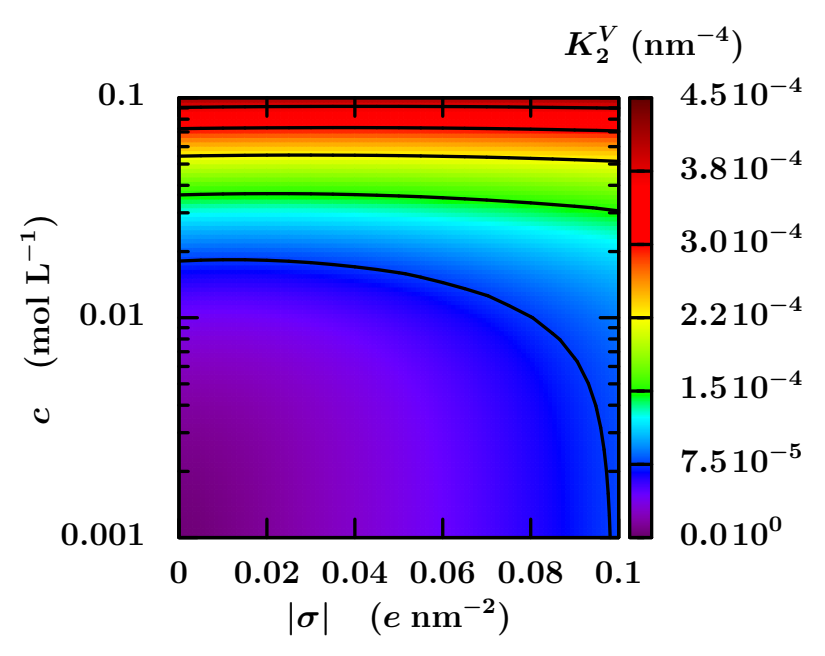

FIG. 9: (Color online) Macroscopic electrical conductance $K_{2}^{V}$ (in $\mathrm{nm}^{-4}$ ) as a function of the salt concentration $c$ in the reservoirs in equilibrium with the charged porous material, and of the surface charge density $\sigma$ of the channels.

the microscopic ones). Therefore, we now turn to the effect of the diameter distribution on the macroscopic coefficients. We focus here on the permeability $K_{0}^{P}$ and on the electro-osmotic coefficient $K_{0}^{V}$. The numerical study in this section is performed for a surface charge density $\sigma=-0.05 e \mathrm{~nm}^{-2}$.

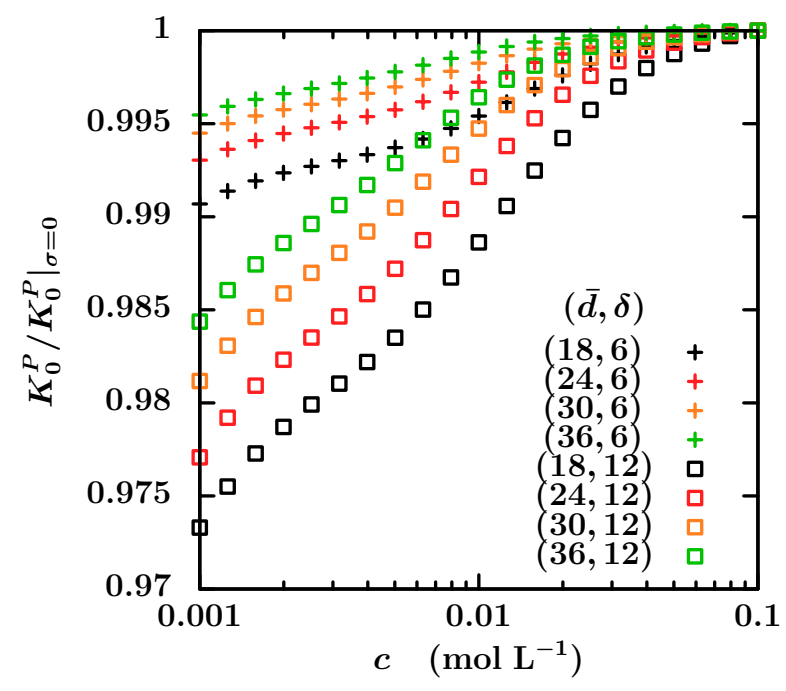

FIG. 10: (Color online) Macroscopic permeability, normalized by the permeability for neutral channels $(\sigma=0)$, as a function of the salt concentration $c$, for diameter Weibull distributions with various means and standard deviations $(\bar{d}, \delta)$. The symbols correspond to $\delta=6 \mathrm{~nm}(+)$ and $\delta=12 \mathrm{~nm}(\square)$. The different colors indicate the mean diameter $\bar{d}: 18 \mathrm{~nm}$ (black), $24 \mathrm{~nm}$ (red), $30 \mathrm{~nm}$ (orange) and $36 \mathrm{~nm}$ (green).
As mentioned above, the permeability of the charged network depends on the salt concentration in the reservoir, as a result of a balance between different types of local fluxes. This contrasts with the case of neutral walls. In Fig. 10 we illustrate the variations of the macroscopic permeability for $\sigma=-0.05 e \mathrm{~nm}^{-2}$ with the salt concentration $c$ in the reservoirs, for different diameter distributions. The decrease of the macroscopic permeability due to local electrokinetic flows is more pronounced at low salt concentration. In addition, in this regime this decrease is larger for smaller average diameters $\bar{d}$ and, for a given $\bar{d}$, for larger standard deviation $\delta$, i.e. in the presence of smaller channels, where electrokinetic effects are more important.

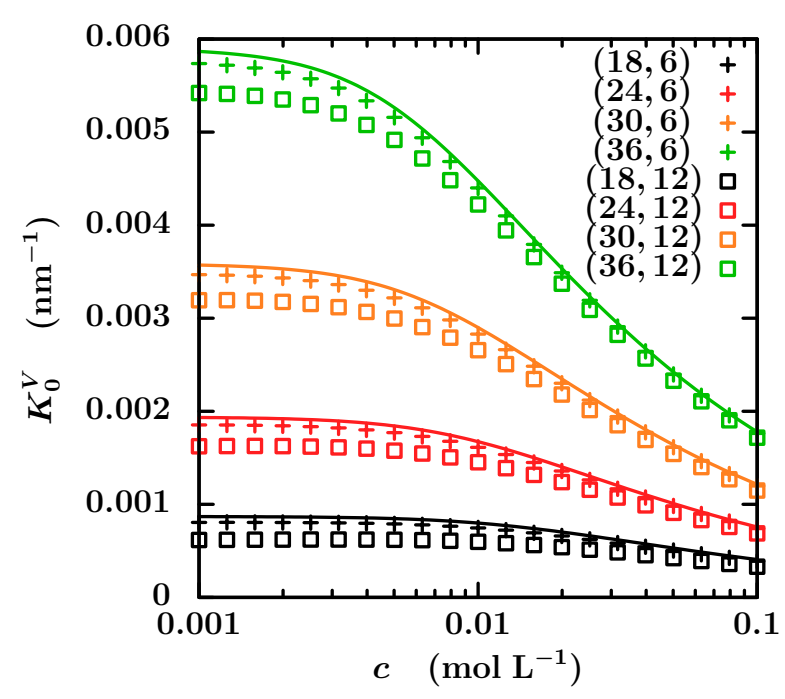

FIG. 11: (Color online) Macroscopic electro-osmotic coefficient as a function of the salt concentration. The solid lines show the result for a single channel with diameter $d=\bar{d}$, see Eq. (7). The same symbols and colors as in Fig. 10 are used.

The effect of the diameter distribution on the macroscopic electro-osmotic coefficient $K_{0}^{V}$ is illustrated in Fig. 11. $K_{0}^{V}$ decreases with increasing salt concentration and decreasing average diameter, as for a single channel of diameter $d=\bar{d}$, also indicated on the figure. $K_{0}^{V}$ also decreases as the standard deviation $\delta$ of the distribution increases, as a result of the presence of a larger number of smaller channels, where electrokinetic effects (which may mitigate the response to the impose macroscopic gradient) are more pronounced. This is consistent with the results of the previous section. Nevertheless, the behaviour of the electro-osmotic coefficient of the complex networks is semi-quantitatively described by that for the corresponding channel with the average diameter. 


\section{Discussion}

The present PNM approach thus allows to demonstrate the combined effect of the coupling between different types of fluxes and the heterogeneity of the sample. For coefficients which already depend strongly on the surface charge density and salt concentration on the channel scale, the macroscopic coefficient exhibit the same dependence on the sample scale. Some qualitative differences may nevertheless arise for large surface charges and low salt concentrations. The effects are more important for coefficients whose dependence on the surface charge and salt concentration is limited, or absent (permeability), on the channel scale.

The overall effect of electrokinetic couplings, which are more pronounced in smaller, more highly charged channels and at lower salt concentrations, is to reduce the macroscopic transport coefficients, compared to the microscopic ones for an average channel. This is consistent with Le Chatelier's principle, as fluxes will locally generate coupled induced flows mitigating the effect of the macroscopic gradient. This underlines the fact that the coefficients of the homogenized transfer matrix $K$ are not simply the homogenized version of the coefficients of the microscopic one, $g$. The upscaled, transport matrix remains however symmetric, in agreement with Onsager's theory, as observed by Brunet and Ajdari from general symmetry arguments [43] or using the mathematical tools of homogenization theory [18 20].

Let us finally comment on the connection between the present model and homogenization. As already mentioned in Sec. II the pores enter in the present model only via the pore variables $P, C$ and $V$. The macroscopic transfer matrix is then determined by ensuring the conservation of the various species $\left(\nabla \cdot q_{\alpha}=0\right)$ at the nodes of the network. Therefore, the present algorithm could also be seen as a discretization of the following continuous problem :

$$
\nabla \cdot\left(g_{\alpha}^{\gamma}(F) \nabla F_{\gamma}\right)=0
$$

with $\{\alpha, \gamma\} \in\{0,1,2\}, F_{0}=P(\mathbf{r}), F_{1}=C(\mathbf{r}), F_{2}=V(\mathbf{r})$ and using Einstein's notation for the implicit sum over the index $\gamma$. The coupling matrix $g(\mathbf{r})$ depends on the value of the fields $F(\mathbf{r})$ via the local Donnan equilibrium. The fluxes $q_{\alpha}(\mathbf{r})$ are then analogous to the average over the small scale model (which we took for simplicity as an infinite cylindrical channel) in the homogenization approach. More interestingly, we can make an analogy between the pore variables and the equivalent bulk variables which were introduced as the natural variables for upscaling in the homogenization approach of Moyne and Murad [18, 19]. How far this analogy can be pushed remains however to be determined.

\section{CONCLUSION}

We proposed a Pore Network Model for the description of the electrokinetic transport properties of charged porous media. This PNM allows to consider the response of heterogeneous samples to external pressure, salt concentration and electric potential gradients. In order to illustrate the interest of this approach, we used simple expressions for the microscopic transport coefficients, based on the Poisson-Nernst-Planck equations in cylindrical channels with fixed surface charge density. We investigated numerically the effect of the surface charge density, salt concentration and channel size distribution on the macroscopic transport coefficients.

The symmetry of the transport matrix is preserved by the present upscaling method, as required from Onsager's theory. The coefficients of this matrix qualitatively behave, in general, as their microscopic counterpart for a channel with the average diameter. However, the combined effects of electrokinetic couplings on the local scale and of heterogeneity result into a decrease of the overall transport coefficients, in accordance with Le Chatelier's principle. This decrease, as well as other qualitative differences which may arise when the microscopic coefficients depend weakly on the surface charge and salt concentration, is more pronounced for large surface charge densities and low salt concentrations, since electrokinetic couplings are stronger in these cases, even at the channel scale. In addition, for a given average diameter, more heterogeneous samples result in stronger effects, due to the presence of smaller pores, in which electrokinetic effects are more pronounced. Overall, the coupling between the complex pore structure of porous media and electrokinetic effects underlines the limitations of approaches based on idealized geometries (single slit pore or cylindrical channel) parametrized directly from the experimental macroscopic properties.

The PNM approach presented here is very flexible and can be straightforwadly enhanced by improving the description of transport on the channel scale. For large surface charges, the analytical expressions used here, which are based on the linearization of the Poisson-Boltzmann equation, are not accurate, but the solution of the nonlinear version can be obtained numerically, as done in [40] in the case considered here or using various mesoscale simulation strategies for electrokinetic effects [49, 50. Osmotic effects could be introduced on the basis of descriptions explicitely considering the radial concentration profiles inside the channel [14, 51]. It is also well know that the PNP model is not valid for too large salt concentrations and too small channels, where the discrete nature of the ions and solvent becomes relevant. Such limitations can be overcomed using improved liquid state theories, e.g. the mean spherical approximation [52, 53] or the Fundamental Measure Theory 54, or by treating 
the electrolyte as a confined mixture 55 57. In principle, for diameters in the nanometer range, it is even possible to determine the channel transfer matrix using molecular dynamics simulations, to capture e.g. the effect of slip at the solid-fluid interface 51, 58. The interest of molecular simulations for the simulation of electrokinetic effects has recently been reviewed [59. In the particular case of clay minerals, which were used here as a guide for numerical applications, such simulations have demonstrated the importance of accounting for slip at the surface to correctly describe the hydrodynamic and electro-osmotic flows 60 62.

Other possible extensions of the proposed approach, this time at the pore network level, include the assignment of finite sizes to the pores, following e.g. the approach proposed in 46]. It remains however to determine how to parametrize such an extended model, which in addition introduces short-range spatial between the pore and channel diameters. Similarly, a strong assumption of the model introduced here is the neglect of the contribution of the interfaces between channels and pores to the overall electrokinetic transport. One way to improve the description would be to determine their effect from simulations including these interfaces explicitly, as done using a mesoscopic model by Marconi et al. to derive effective one-dimensional transport equations [45, and to introduce the results in modified conductance matrices for the channels. The present model can now be used to determine the properties of a given sample, if sufficient experimental data is available for the parametrization of the model, or to investigate the properties of generic materials, in particular percolation properties [37, 47, 63] for the various types of fluxes.

\section{Acknowledgments}

A.O. acknowledges financial support from Andra.

* Electronic address: amael.obliger@gmail.com

[1] R. R. Thompson, Geophysics 1, 327 (1936)

[2] S. R. Pride and M. W. Haartsen, The Journal of the Acoustical Society of America 100, 1301 (1996).

[3] H. Mizutani, T. Ishido, T. Yokokura, and S. Ohnishi, Geophysical Research Letters 3, 365 (1976).

[4] D. T. Luong and R. Sprik, ISRN Geophysics 2013, 1 (2013)

[5] C. K. Haluska, K. A. Riske, V. Marchi-Artzner, J. M. Lehn, R. Lipowsky, and R. Dimova, Proc. Nat. Acad. Sci. 103, 15841 (2006).

[6] R. Dimova, K. A. Riske, S. Aranda, N. Bezlyepkina, R. L. Knorr, and R. Lipowsky, Soft Matter 3, 817 (2007).
[7] D. Lacoste, G. Menon, M. Z. Bazant, and J. F. Joanny, Europ. Phys. J. E 28, 243 (2009).

[8] C. C. Harrell, S. B. Lee, and C. R. Martin, Analytical Chemistry 75, $6861(2003)$

[9] S. Song, A. K. Singh, T. J. Shepodd, and B. J. Kirby, Analytical Chemistry 76, 2367 (2004).

[10] D. J. Harrison, K. Fluri, K. Seiler, Z. Fan, C. S. Effenhauser, and A. Manz, Science 261, 895 (1993)

[11] S. van Dorp, U. F. Keyser, N. H. Dekker, C. Dekker, and S. G. Lemay, Nat. Phys. 5, 347 (2009).

[12] G. M. Skinner, M. van den Hout, O. Broekmans, C. Dekker, and N. H. Dekker, Nano Letters 9, 2953 (2009).

[13] A. Ajdari, Applied Physics A 75, 271 (2002).

[14] A. Siria, P. Poncharal, A.-L. Biance, R. Fulcrand, X. Blase, S. T. Purcell, and L. Bocquet, Nature 494, 455 (2013)

[15] S. Pride, Physical Review B 50, 15678 (1994).

[16] A. Revil and P. Leroy, Journal of Geophysical Research: Solid Earth 109, B03208 (2004)

[17] J. R. Looker and S. L. Carnie, Transport in porous media 65, 107 (2006)

[18] C. Moyne and M. A. Murad, Transport in Porous Media 62, $333(2006)$

[19] C. Moyne and M. A. Murad, Transport in Porous Media 63, 13 (2006).

[20] G. Allaire, A. Mikelic, and A. Piatnitski, Journal of Mathematical Physics 51, 123103 (2010)

[21] E. Bresler, Water Resources Research 9, 975 (1973)

[22] J. Gonçalvès, G. de Marsily, and J. Trémosa, Earth and Planetary Science Letters 339340, 1 (2012)

[23] D. Coelho, M. Shapiro, J. F. Thovert, and P. M. Adler, Journal of colloid and interface science 181, 169 (1996)

[24] S. Marino, M. Shapiro, and P. Adler, Journal of Colloid and Interface Science 243, 391 (2001)

[25] A. K. Gupta, D. Coelho, and P. M. Adler, Journal of Colloid and Interface Science 303, 593 (2006)

[26] I. Fatt, Petrol Trans AIME , 144159 (1956).

[27] P.-E. Oren, S. Bakke, and O. Arntzen, SPE Journal 3 (1998), 10.2118/52052-PA

[28] M. J. Blunt, Current Opinion in Colloid \& Interface Science 6, 197 (2001).

[29] M. I. J. van Dijke and K. S. Sorbie, Physical Review E 66, $046302(2002)$

[30] S. Békri, C. Laroche, and O. Vizika, in SCA, Vol. 35 (2005) p. 2005.

[31] C. Laroche and O. Vizika, Transport in Porous Media 61, 77 (2005).

[32] J. Ochi and J.-F. Vernoux, Transport in Porous Media 37, 303 (1999)

[33] C. Lu and Y. C. Yortsos, Physical Review E 72, 036201 (2005).

[34] L. Algive, S. Békri, and O. Vizika, SPE Journal 15 (2010), 10.2118/124305-PA

[35] A. Raoof, S. M. Hassanizadeh, and A. Leijnse, Vadose Zone Journal 9, $624(2010)$

[36] C. Varloteaux, M. T. Vu, S. Békri, and P. M. Adler, Physical Review E 87, 023010 (2013).

[37] Y. Bernabé, Journal of Geophysical Research: Solid Earth 103, 2082720841 (1998)

[38] A. Revil, L. M. Cathles, S. Losh, and J. A. Nunn, Journal of Geophysical Research: Solid Earth 103, 2392523936 (1998)

[39] C. L. Rice and R. Whitehead, The Journal of Physical 
Chemistry 69, 4017 (1965).

[40] A. Obliger, M. Duvail, M. Jardat, D. Coelho, S. Békri, and B. Rotenberg, Physical Review E 88, 013019 (2013).

[41] A. Gupta, D. Coelho, and P. Adler, Journal of colloid and interface science 319, 549 (2008)

[42] D.L. Johnson, J. Koplik, and L.M. Schwartz, Physical Review Letters 57, 2564 (1986).

[43] E. Brunet and A. Ajdari, Physical Review E 69, 016306 (2004).

[44] S. Gravelle, L. Joly, F. Detcheverry, C. Ybert, C. CottinBizonne, and L. Bocquet, Proceedings of the National Academy of Sciences 110, 16367 (2013).

[45] U. M. B. Marconi, S. Melchionna, and I. Pagonabarraga, The Journal of Chemical Physics 138, 244107 (2013)

[46] I. Chatzis and F. Dullien, Journal of Canadian Petroleum Technology (1977), 10.2118/77-01-09

[47] B. Berkowitz and R. P. Ewing, Surveys in Geophysics 19, 2372 (1998).

[48] H. Nguyen, Z. Bai, E. C. Dogrul, T. N. Kadir, C. F. Brush, and F. I. Chung, XIX International Conference on Water Resources (2012)

[49] B. Rotenberg, I. Pagonabarraga, and D. Frenkel, Faraday Discussions 144, 223 (2009).

[50] I. Pagonabarraga, B. Rotenberg, and D. Frenkel, Phys. Chem. Chem. Phys. 12, 9566 (2010).

[51] A. Ajdari and L. Bocquet, Physical Review Letters 96, $186102(2006)$

[52] J.-F. Dufrêche, O. Bernard, S. Durand-Vidal, and
P. Turq, The Journal of Physical Chemistry B 109, 9873 (2005)

[53] M. Jardat, J.-F. Dufrêche, V. Marry, B. Rotenberg, and P. Turq, Physical Chemistry Chemical Physics 11, 2023 (2009)

[54] D. Gillespie, W. Nonner, and R. S. Eisenberg, Journal of Physics: Condensed Matter 14, 12129 (2002)

[55] U. Marini Bettolo Marconi and S. Melchionna, The Journal of Chemical Physics 131, 014105 (2009)

[56] S. Melchionna and U. Marini Bettolo Marconi, Europhysics Letters 95, 44002 (2011).

[57] U. Marini Bettolo Marconi and S. Melchionna, Langmuir 28, 13727 (2012).

[58] L. Bocquet and J.-L. Barrat, Soft Matter 3, 685 (2007)

[59] B. Rotenberg and I. Pagonabarraga, Molecular Physics 111, 827 (2013).

[60] A. Botan, B. Rotenberg, V. Marry, P. Turq, and B. Noetinger, The Journal of Physical Chemistry C 115, 16109 (2011)

[61] A. Botan, V. Marry, B. Rotenberg, P. Turq, and B. Noetinger, The Journal of Physical Chemistry C 117, 978 (2013).

[62] A. Botan, V. Marry, B. Rotenberg, P. Turq, and B. Noetinger, The Journal of Physical Chemistry C 117, 20376 (2013).

[63] A. H. Thompson, A. J. Katz, and R. A. Raschke, Physical Review Letters 58, 29 (1987) 